\title{
CICLO DE CONVERSIÓN DEL EFECTIVO Y SU INCIDENCIA EN LA LIQUIDEZ DE UNA EMPRESA INDUSTRIAL
}

\author{
CASH CONVERSION CYCLE AND ITS IMPACT ON THE LIQUIDITY OF AN \\ INDUSTRIAL COMPANY
}

Patrick Ademir Diaz Cuenca Universidad Peruana Unión Lima, Perú

ORCID: https://orcid.org/0000-0002-0772-9940 Correo electrónico: patrickademir@upeu.edu.pe

Jesabel Jesús Ramón Martínez Universidad Peruana Unión Lima, Perú ORCID: https://orcid.org/0000-0003-2148-0907 Correo electrónico: jesabel.ramon@upeu.edu.pe

\section{RESUMEN}

Objetivo: Establecer la incidencia del ciclo de conversión del efectivo en la liquidez de la empresa industrial Damar G\&L S.A.C de Lima, durante los periodos 2016 al 2019. Método: El estudio presentó un enfoque cuantitativo con un alcance correlacional de corte transversal, utilizando un diseño no experimental. El método aplicado para la recolección de los datos fue el análisis documental; como población de estudio se utilizó datos de los registros contables de cuentas por cobrar, inventarios y cuentas por pagar de la empresa, estructurados en 48 estados financieros mensualizados. Resultados: Los resultados evidencian una relación mínima no significativa entre el ciclo de conversión del efectivo y la liquidez (Rho $=.075, p=.614$ ) correspondiente a los periodos en estudio; asimismo, los indicadores de la variable independiente, periodo de rotación de cuentas por cobrar, periodo de rotación de inventarios y periodo de rotación de cuentas por pagar, muestran una relación débil ( $R h o=.201,-.267, .086)$ en la prueba ácida, con un nivel de significancia mayor al $5 \%$. Conclusión: Se concluye que durante el periodo comprendido 2016 al 2019 de la empresa en estudio, el ciclo de conversión del efectivo no incide directamente en la liquidez.

Palabras clave: Ciclo de efectivo; liquidez; rotación; efectivo; empresa.

\begin{abstract}
Objective: To establish the impact of the cash conversion cycle on the liquidity of the industrial company Damar G\&L S.A.C of Lima, during 2016 to 2019. Method: The study presented a quantitative approach with a range of cross-sectional correlational, using a non-experimental design. The method used to collect the data was the documentary analysis. As the study population, this research used data from the accounting records of accounts receivable, inventories, and the company's payable accounts, in which 48 financial monthly statements were structured. Results: The results show that there is a minimal non-significant relationship between the cash conversion cycle and liquidity (Rho $=.075, \mathrm{p}=$ .614) corresponding to the periods under study. Furthermore, the independent variable's indicators, accounts receivable turnover period, inventory turnover period, and accounts payable turnover period, show a weak relationship (Rho $=.201,-.267, .086)$ in the acid test, with a significance level greater than $5 \%$. Conclusion: It is concluded that, during the 2016-2019 period of the company under review, the cash conversion cycle does not directly affect its liquidity.
\end{abstract}

Keywords: Cash cycle; liquidity; rotation; cash; company.

(c) Los autores. Este artículo es publicado por la revista Quipukamayoc de la Facultad de Ciencias Contables, Universidad Nacional Mayor de San Marcos. Este es un artículo de acceso abierto, distribuido bajo los términos de la licencia Creative Commons Atribución 4.0 Internacional (CC BY 4.0) [https://creativecommons.org/licenses by/4.0/deed.es] que permite el uso, distribución y reproducción en cualquier medio, siempre que la obra original sea debidamente citada de su fuente original. 


\section{INTRODUCCIÓN}

Actualmente, el mundo empresarial vive en constantes cambios, las empresas necesitan mantener la eficiencia y eficacia en cada una de sus operaciones mercantiles; por ello, se proyectan constantemente en mejorar sus actividades económicas, trayendo consigo óptimos resultados que se reflejan en la generación de utilidades. Una empresa que administra correctamente el dinero puede disponer de efectivo para solventar cualquier eventual necesidad que requiera de una rápida solución. Por eso, es vital que en la gestión de los activos corrientes se logre comprender el ciclo de conversión del efectivo de una empresa, pues este mide el tiempo que se necesita para que una inversión se convierta en efectivo, siendo producto de sus operaciones (Gitman y Zutter, 2012). Asimismo, si una empresa adopta una correcta aplicación de estrategias en la administración del dinero, no presentará problemas de liquidez y cubrirá sus necesidades financieras y, a su vez, tendrá el tiempo necesario en días para trabajar con suficiente capital propio (García, Galarza y Altamirano, 2017).

Samper (2015) hace referencia al ciclo de conversión del efectivo como ciclo de explotación o periodo medio de maduración (PMM), donde menciona que una organización es más efectiva y eficiente cuando tiene un periodo medio de maduración corto; es decir, cuando una empresa tiene un PMM menor tendrá mayores rotaciones o ciclos, pero si su ciclo es bajo el PMM será más alto, causando que el volumen de su financiamiento se eleve al igual que sus costos.

Asimismo, Jiménez, Rojas y Ospina (2013) mencionan la importancia que tiene el ciclo de caja o ciclo de efectivo dentro de una organización, porque si este se maneja de manera inadecuada, generará iliquidez y la empresa incumplirá con el pago de sus obligaciones a corto plazo. Por ello, el cálculo y manejo del ciclo de caja ocasiona problemas y preocupación a la gerencia, no solo porque refleja la gestión financiera, sino que también impacta en la información presentada en los estados financieros.

La Norma Internacional de Contabilidad $\mathrm{N}^{\mathrm{O}} 1$ (2006), Presentación de Estados Financieros, hace referencia al ciclo de conversión del efectivo (CCE) y su importancia a la hora de elaborar y presentar informes financieros necesarios para evaluar el estado económico y financiero de una empresa. La norma refiere lo siguiente:

El ciclo normal de la operación de una entidad es el periodo de tiempo que transcurre entre la adquisición de los activos materiales, que entran en el proceso productivo, y la realización de los productos en forma de efectivo o equivalentes al efectivo. El activo corriente incluye activos (tales como inventarios y deudores comerciales) que se van a vender, consumir y realizar, dentro del ciclo normal de la operación, incluso cuando los mismos no se esperen realizar dentro del periodo de doce meses desde la fecha de balance. (párr. 59)

Por ello, el ciclo de conversión del efectivo es una herramienta fundamental en el área de la contabilidad y las finanzas para la evaluación de la situación financiera de toda organización, ya que da a conocer si una empresa tiene suficiente flujo de efectivo para recuperar y reestablecer el capital de trabajo; pues es fundamental que toda organización pueda generar su propio efectivo a fin de contrarrestar cualquier financiamiento a corto plazo (Jiménez, Rojas y Ospina, 2013). Si el ciclo de conversión del efectivo es mínimo, disminuyen los recursos que requiere el capital de trabajo y su aumento año tras año (Arcos y Benavides, 2008). De esta manera, el ciclo de conversión del efectivo se convierte en el pilar fundamental para toda entidad porque le da valor e incrementa las utilidades de los socios; además, tiene como finalidad dar a conocer si la gestión de cobranza a clientes, la rotación de existencias y el pago a proveedores están siendo administradas de manera correcta y eficiente (Ramos y Sandoval, 2019).

Es trascendental que en la gestión eficaz del capital de trabajo se tomen las mejores decisiones en relación con el desarrollo y crecimiento de una organización, porque cuando un país enfrenta problemas graves de financiamiento, esto impacta directamente en la estructura financiera de toda empresa. Por lo tanto, hoy en día, la administración del efectivo se ha convertido en todo un desafío con el fin de encontrar la estabilidad y prosperidad empresarial, de no llegar a ella, la incapacidad en la administración generaría una reducción en la rentabilidad y conllevaría a la empresa a una severa crisis financiera (Murtala y Sani, 2016).

Asimismo, la gestión de la liquidez juega un papel crucial en el éxito financiero de una organización, teniendo en cuenta que si una empresa no gestiona eficientemente a sus activos y pasivos, no podrá pagar a sus proveedores en el momento del vencimiento de sus obligaciones (Al-Shubiri y Mohammad, 2013). Además, como indican Lyroudi y Mccarty (1993), el pago anticipado de las deudas y obligaciones de corto plazo genera problemas de inestabilidad en muchas de las empresas porque estas recurrirán al financiamiento de entidades externas, y si estas son pequeñas o no cuentan con suficiente capacidad financiera, obtendrán créditos a costos elevados por su propensión a asumir riesgos; de ese modo, no cumplirán con las demandas de crecimiento impuestas por el mercado empresarial.

La inseguridad que existe en cuanto a las cobranzas y los pagos en gran parte de las empresas que pertenecen a los 
países de América Latina, es generada porque invierten exageradamente en el capital de trabajo a causa de una ineficiente proyección de ventas. Por otro lado, existe una relación directa en cuanto a la inversión a corto plazo que realizan las empresas latinoamericanas, pues esto explicaría el exceso de inversión del activo corriente. De este modo, el crecimiento de las organizaciones latinoamericanas se debe, en mucho de los casos, a que se invierte más en el corto plazo que en periodos de largo plazo (Mongrut y Wong, 2005, citado por Mongrut, Fuenzalida, Cubillas y Cubillas, 2014).

La situación de riesgo de liquidez de las empresas peruanas ha mejorado considerablemente desde el año 2015, debido a que a fines del año 2018 estas tenían mayor posibilidad de cubrir sus pasivos de corto plazo. Sin embargo, a pesar de haber mejorado, aún existe un riesgo elevado en la liquidez a diferencia de otras empresas de América Latina. La dependencia al refinanciamiento de las deudas $\mathrm{u}$ obligaciones a corto plazo, genera que las organizaciones no tengan accesibilidad a la liquidez en periodos de crisis financieras (García, 2019). Por otra parte, en comparación a las demás naciones que conforman la Alianza del Pacífico, las empresas peruanas cumplen con sus deudas a corto plazo, es decir, sus activos corrientes cubren a los pasivos corrientes hasta 1.3 veces (García, 2018).

Durante el periodo de investigación, Damar G\&L S.A.C., empresa industrial dedicada a la fabricación de productos de plástico, ha presentado problemas originados por la inadecuada gestión de sus activos y pasivos, pues ha mantenido una inestabilidad en los días de recuperación del efectivo por los créditos otorgados a sus clientes, como también en los reembolsos realizados a los proveedores por la compra de materia prima e insumos. Este hecho perjudica el buen desempeño de una gestión eficiente en lo que respecta a las entradas y salidas de dinero; por cuanto, los días de cobro a los clientes y los días de pago a los proveedores no se establecen con precisión. Asimismo, no ha tenido un control en los inventarios, puesto que adquiere materia prima con la finalidad de producir pero sin establecer una proyección fija de ventas, llegando a tener un exceso de existencias al cierre de cada periodo y generando problemas de liquidez e incrementando sus deudas. En este entorno, surge el interés por establecer si el ciclo de conversión del efectivo incide en la liquidez de la empresa industrial durante los años de estudio 2016 al 2019.

Si bien para la medición de la liquidez se puede utilizar cada uno de sus indicadores, es importante mencionar que en la evaluación de los activos líquidos de la empresa, se determinó que cuenta con inventarios no corrientes; por tal motivo, para este estudio se ha utilizado al ratio de prueba ácida, que según Ross, Westerfield y Jordan (2010) el inventario es uno de los activos menos líquidos y que muchas veces en los libros se registran valores no confiables, dado que no se toma en cuenta características como la calidad del inventario. La utilización de este ratio de liquidez permitirá conocer si la empresa cuenta con la solidez financiera para cubrir sus deudas y asumir riesgos de iliquidez, excluyendo al valor realizable del activo corriente.

Se han analizado las siguientes investigaciones que se asocian al estudio del ciclo de conversión del efectivo y su incidencia en la liquidez.

Culqui (2011) ha realizado una investigación sobre la incidencia del ciclo de caja en la liquidez de la empresa Construcciones Civil-Hidráulica Culqui Ninacuri, encontrando que la empresa realiza análisis financieros empíricos para determinar la liquidez y carece de capital propio, pues la liquidez que mantiene es por créditos de terceros, considerando que la mayoría de sus cuentas por cobrar son a largo plazo.

Graziano (2016) ha estudiado la relación del ciclo de conversión del efectivo en la inversión y liquidez en las empresas picadoras de piedra caliza del Municipio Rosario de Perijá, encontrando la existencia de una relación alta y positiva entre el ciclo de conversión del efectivo y la liquidez. Además, que aunque las empresas tienen conocimiento del ciclo de conversión del efectivo y la liquidez, se limitan en aplicar nuevas políticas y a generar nuevos conocimientos para realizar un correcto registro de los periodos de cobranza, inventarios y pagos para de esa manera mantener una eficiente administración del dinero.

Molina (2016) ha estudiado la relación entre el ciclo de conversión del efectivo y la liquidez de la empresa Lácteos Piamonte S.A.C. durante el período 2016, encontrando que existe una correlación significativa entre el ciclo de conversión del efectivo y la liquidez de la empresa en estudio.

Castro (2017) ha realizado una investigación sobre el ciclo de conversión del efectivo y la liquidez de la empresa Electro Frío, encontrando que la entidad tiene un déficit en el control de sus inventarios y el periodo de cobro es muy alto a comparación de su periodo de pago que es mucho menor, dado que brinda créditos a clientes morosos y las deudas a proveedores son cubiertas con el capital propio de la empresa.

Sanjines (2019) ha realizado un estudio sobre la aplicación de las razones financieras de actividad en una organización: gestión y repercusiones en la liquidez de la empresa COMPLEX-U1, encontrando que tiene problemas a causa de los retrasos en la recuperación de la cartera de cobranza y la rotación de los inventarios es baja, lo cual se evidencia en un déficit de liquidez; por ello, no puede solventar sus deudas y obligaciones de corto plazo. 
Desde el contexto de estas investigaciones, se plantea el objetivo de establecer la incidencia del ciclo de conversión del efectivo en la liquidez de la empresa industrial Damar G\&L S.A.C durante los periodos 2016-2019. En ese sentido, la hipótesis planteada responde a la pregunta: El ciclo de conversión del efectivo incide significativamente en la liquidez de la empresa industrial Damar G\&L S.A.C. durante los periodos 2016-2019.

\section{MATERIALES Y MÉTODOS}

La investigación presentó un enfoque cuantitativo con un alcance correlacional de corte transversal, utilizando un diseño no experimental, porque se realizó sin la manipulación de las variables, siendo que, solo se observan los hechos existentes para poder analizarlos en su contexto o estado natural (Hernández, Fernández y Baptista, 2014).

Asimismo, el estudio estuvo conformado por una unidad de análisis, identificada por la empresa industrial Damar G\&L S.A.C. $y$, teniendo en cuenta que el estudio tiene una duración de cuatro años, correspondiente a los periodos 2016 al 2019, se revisaron los registros contables y balances generales determinados por 48 periodos mensuales, iniciando desde el mes de enero del 2016 hasta el mes de diciembre del 2019. Los datos fueron estructurados en informes financieros que obedecen a las necesidades específicas de la investigación.

Además, los instrumentos utilizados fueron fichas de recolección de datos, estructuradas para recopilar de forma detallada ratios financieros mensualizados tanto del ciclo de conversión del efectivo como el de liquidez. Los datos recabados fueron procesados, analizados y validados con el software estadístico SPSS Statistics 25. Para el análisis de la distribución normal de los datos, se aplicó la prueba de normalidad de Shapiro-Wilks (datos menores a 50); determinando que los datos cuentan con una distribución no normal. Por lo tanto, se usó el coeficiente de correlación Rho de Spearman para la aplicación de la prueba de hipótesis.

\section{RESULTADOS}

En la tabla 1, se muestra los resultados del análisis de los ratios financieros de la empresa industrial Damar G\&L S.A.C durante los periodos de estudio 2016 al 2019. Con la obtención de los ratios, se pretende establecer la existencia de una relación entre las variables de estudio como también en sus indicadores, y al mismo tiempo determinar la gestión financiera de la empresa. Cabe resaltar que, en el mes de abril del año 2017 la empresa tuvo un bajo nivel de ventas (0.3\%), debido a que, en marzo del mismo año, un desastre natural devastó a la empresa dejándola completamente inoperativa; en consecuencia, los resultados del mes de abril correspondientes a los ratios periodo de rotación de cuentas por cobrar y periodo de rotación de inventarios, se calcularon tomando como base una media móvil de ventas, con el fin de no distorsionar los resultados de la investigación.

Se observa, en la tabla 2, los resultados del análisis descriptivo de la empresa industrial Damar G\&L S.A.C. durante los periodos de estudio 2016 al 2019.

El periodo de rotación de cuentas por cobrar tiene un rango de 316.74 días, donde el valor mínimo del periodo de cobro es de 61.84 días y el máximo es de 378.57 días; con un valor promedio de 149.66 días y una desviación estándar de 70.17 días, lo que refleja una variabilidad del $47 \%$, que confirma la dispersión entre los valores máximo y mínimo. El periodo de rotación de inventarios presenta un rango de 66.63 días, en el que el valor mínimo del periodo de inventarios es de 12.63 días y el máximo es de 79.26 días; con un valor medio de 43.94 días y una desviación estándar de 19.58 días, lo que muestra una variación del $45 \%$, confirmando la amplitud de los datos del rango entre los valores mínimo y máximo. El periodo de rotación de cuentas por pagar cuenta con un rango de 593.77 días, donde el valor menor del periodo de pago es de 35.05 días y el mayor es de 628.82 días; presentando un valor promedio de 164.59 días y una desviación estándar de 120.31 días, lo que resulta en una variabilidad del $73 \%$, que confirma lo amplio del rango según los datos del valor menor y mayor. Asimismo, el ciclo de conversión del efectivo tiene un rango de 614.89 días, siendo que el valor mínimo del ciclo de efectivo es de -430.44 días y el máximo es de 184.45 días; con un valor promedio de 29.01 días y una desviación estándar de 111.62 días, evidenciando una variabilidad del $385 \%$; por consiguiente, debido a que los datos oscilan entre valores positivos y negativos, se refleja una dispersión bastante amplia entre los datos del rango según los valores máximo y mínimo. La prueba ácida presenta un rango de 0.490 , en el que el valor menor de liquidez es de 0.586 y el mayor es de 1.076; con un valor medio de 0.795 y una desviación estándar de 0.098, lo que refleja una variación del $12 \%$, confirmando una extensión del rango según los valores extremos.

El comportamiento del indicador periodo de rotación de cuentas por cobrar se muestra en la figura 1. Se verifica que la empresa industrial durante los periodos 2016 al 2019 muestra una pendiente positiva de 0.7964 , lo que representa un nivel de crecimiento en el promedio de días de cobro. Asimismo, el indicador muestra que si bien en el año 2016 el promedio de días de cobro se ha mantenido en un nivel bajo de 61.84 días, es a partir del mes de marzo del 2017 donde se incrementó, alcanzando un máximo nivel de 378.57 días, del mismo modo, en el año 2018 obtuvo un promedio elevado de 252.18 días de cobranza, ya en el 
Tabla 1

Análisis de los ratios financieros de la empresa Damar GéL S.A.C.

\begin{tabular}{|c|c|c|c|c|c|}
\hline Periodo & $\begin{array}{l}\text { Periodo de rotación de } \\
\text { cuentas por cobrar }\end{array}$ & $\begin{array}{l}\text { Periodo de rotación de } \\
\text { inventarios }\end{array}$ & $\begin{array}{l}\text { Periodo de rotación de } \\
\text { cuentas por pagar }\end{array}$ & $\begin{array}{l}\text { Ciclo de conversión } \\
\text { del efectivo }\end{array}$ & $\begin{array}{l}\text { Prueba } \\
\text { ácida }\end{array}$ \\
\hline 2016-01 & 80.42 & 79.26 & 379.84 & -220.16 & 0.61 \\
\hline 2016-02 & 61.84 & 64.71 & 72.34 & 54.20 & 0.59 \\
\hline 2016-03 & 62.80 & 61.38 & 85.12 & 39.05 & 0.60 \\
\hline 2016-04 & 87.84 & 74.43 & 143.27 & 19.00 & 0.64 \\
\hline 2016-05 & 79.21 & 57.08 & 188.16 & -51.87 & 0.68 \\
\hline 2016-06 & 94.79 & 73.04 & 82.32 & 85.51 & 0.63 \\
\hline 2016-07 & 84.81 & 78.02 & 136.29 & 26.55 & 0.85 \\
\hline 2016-08 & 80.67 & 71.29 & 128.85 & 23.11 & 0.84 \\
\hline 2016-09 & 72.31 & 55.09 & 116.94 & 10.46 & 0.90 \\
\hline $2016-10$ & 98.17 & 73.45 & 118.59 & 53.02 & 0.89 \\
\hline 2016-11 & 78.69 & 62.11 & 145.98 & -5.18 & 0.89 \\
\hline 2016-12 & 95.76 & 62.02 & 137.24 & 20.54 & 0.93 \\
\hline $2017-01$ & 108.23 & 58.22 & 77.94 & 88.51 & 0.89 \\
\hline 2017-02 & 114.46 & 57.73 & 113.85 & 58.34 & 0.83 \\
\hline 2017-03 & 190.10 & 51.63 & 128.57 & 113.16 & 0.82 \\
\hline 2017-04 & 318.53 & 21.25 & 347.15 & -7.37 & 0.78 \\
\hline 2017-05 & 260.19 & 44.85 & 120.59 & 184.45 & 0.74 \\
\hline 2017-06 & 311.13 & 64.72 & 509.57 & -133.72 & 0.78 \\
\hline $2017-07$ & 208.21 & 32.63 & 233.46 & 7.37 & 0.80 \\
\hline 2017-08 & 378.57 & 50.52 & 305.42 & 123.67 & 0.80 \\
\hline 2017-09 & 221.27 & 32.08 & 132.96 & 120.38 & 0.93 \\
\hline $2017-10$ & 165.79 & 22.32 & 121.85 & 66.26 & 0.96 \\
\hline $2017-11$ & 174.10 & 17.22 & 101.03 & 90.29 & 0.96 \\
\hline $2017-12$ & 189.45 & 17.52 & 117.75 & 89.22 & 1.08 \\
\hline 2018-01 & 113.91 & 12.63 & 44.81 & 81.73 & 0.87 \\
\hline 2018-02 & 252.18 & 26.51 & 124.93 & 153.76 & 0.87 \\
\hline 2018-03 & 151.65 & 25.23 & 35.05 & 141.83 & 0.77 \\
\hline 2018-04 & 141.82 & 27.21 & 119.96 & 49.07 & 0.82 \\
\hline 2018-05 & 241.75 & 33.92 & 119.25 & 156.41 & 0.80 \\
\hline 2018-06 & 206.51 & 30.37 & 72.93 & 163.95 & 0.78 \\
\hline 2018-07 & 200.41 & 29.58 & 130.31 & 99.67 & 0.77 \\
\hline 2018-08 & 130.77 & 23.32 & 52.48 & 101.61 & 0.76 \\
\hline 2018-09 & 154.90 & 23.84 & 237.61 & -58.88 & 0.79 \\
\hline $2018-10$ & 136.13 & 21.83 & 57.84 & 100.11 & 0.75 \\
\hline 2018-11 & 106.41 & 23.34 & 74.86 & 54.89 & 0.73 \\
\hline $2018-12$ & 131.78 & 28.58 & 99.61 & 60.75 & 0.75 \\
\hline 2019-01 & 96.65 & 20.27 & 65.37 & 51.55 & 0.78 \\
\hline 2019-02 & 120.79 & 28.77 & 68.48 & 81.08 & 0.78 \\
\hline 2019-03 & 96.92 & 22.56 & 164.91 & -45.43 & 0.84 \\
\hline 2019-04 & 158.06 & 28.35 & 300.26 & -113.84 & 0.87 \\
\hline 2019-05 & 167.87 & 41.74 & 127.66 & 81.95 & 0.79 \\
\hline 2019-06 & 152.03 & 46.35 & 628.82 & -430.44 & 0.82 \\
\hline 2019-07 & 169.36 & 61.05 & 117.21 & 113.20 & 0.75 \\
\hline 2019-08 & 122.98 & 49.27 & 299.21 & -126.95 & 0.78 \\
\hline 2019-09 & 139.64 & 62.90 & 99.04 & 103.50 & 0.70 \\
\hline $2019-10$ & 117.23 & 61.57 & 305.96 & -127.16 & 0.72 \\
\hline 2019-11 & 115.57 & 50.02 & 281.88 & -116.29 & 0.77 \\
\hline 2019-12 & 141.00 & 47.46 & 226.86 & -38.40 & 0.73 \\
\hline
\end{tabular}

Fuente: Elaboración propia 
Tabla 2

Resultados descriptivos de la empresa Damar G\&L S.A.C.

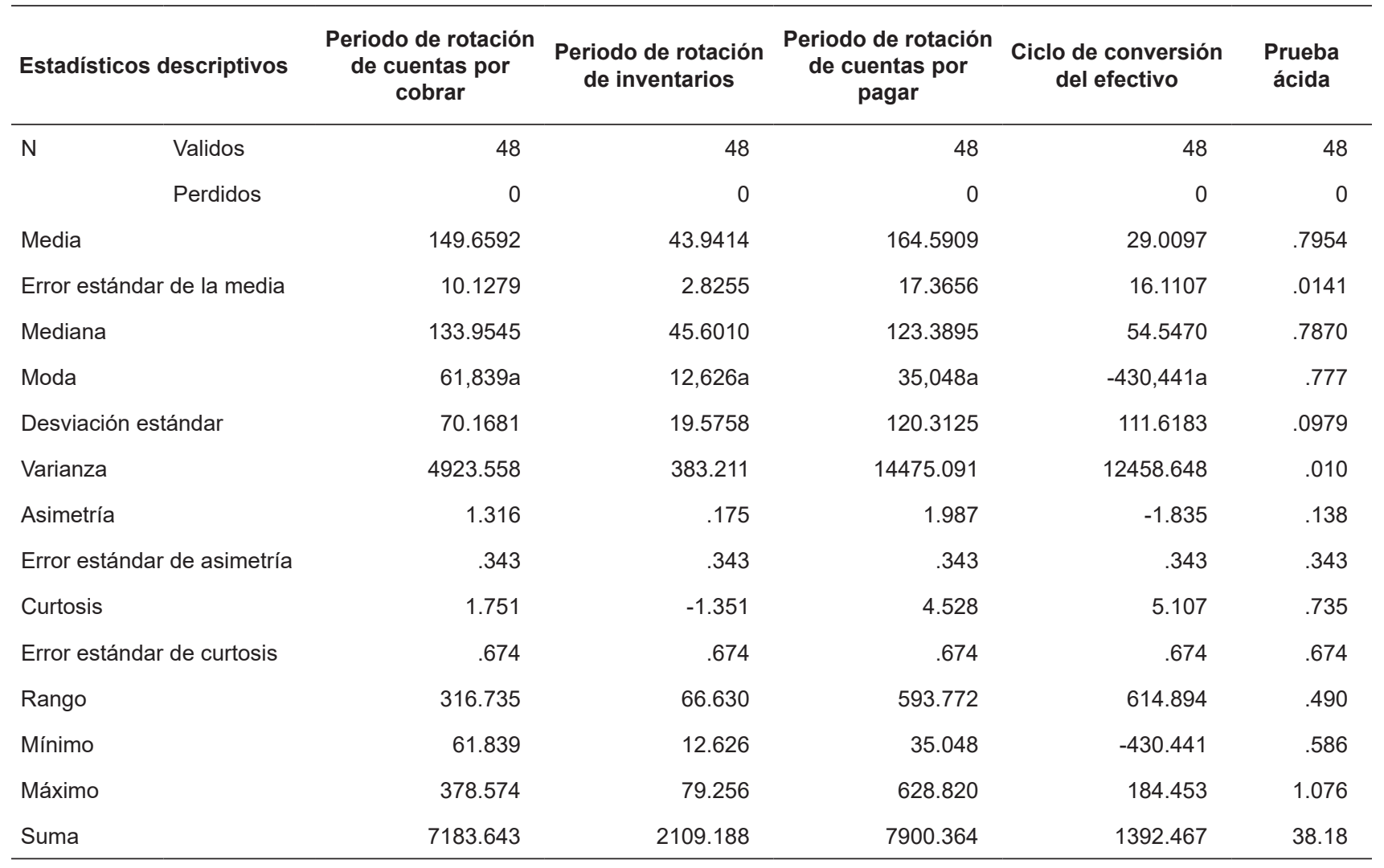

Fuente: Elaboración propia

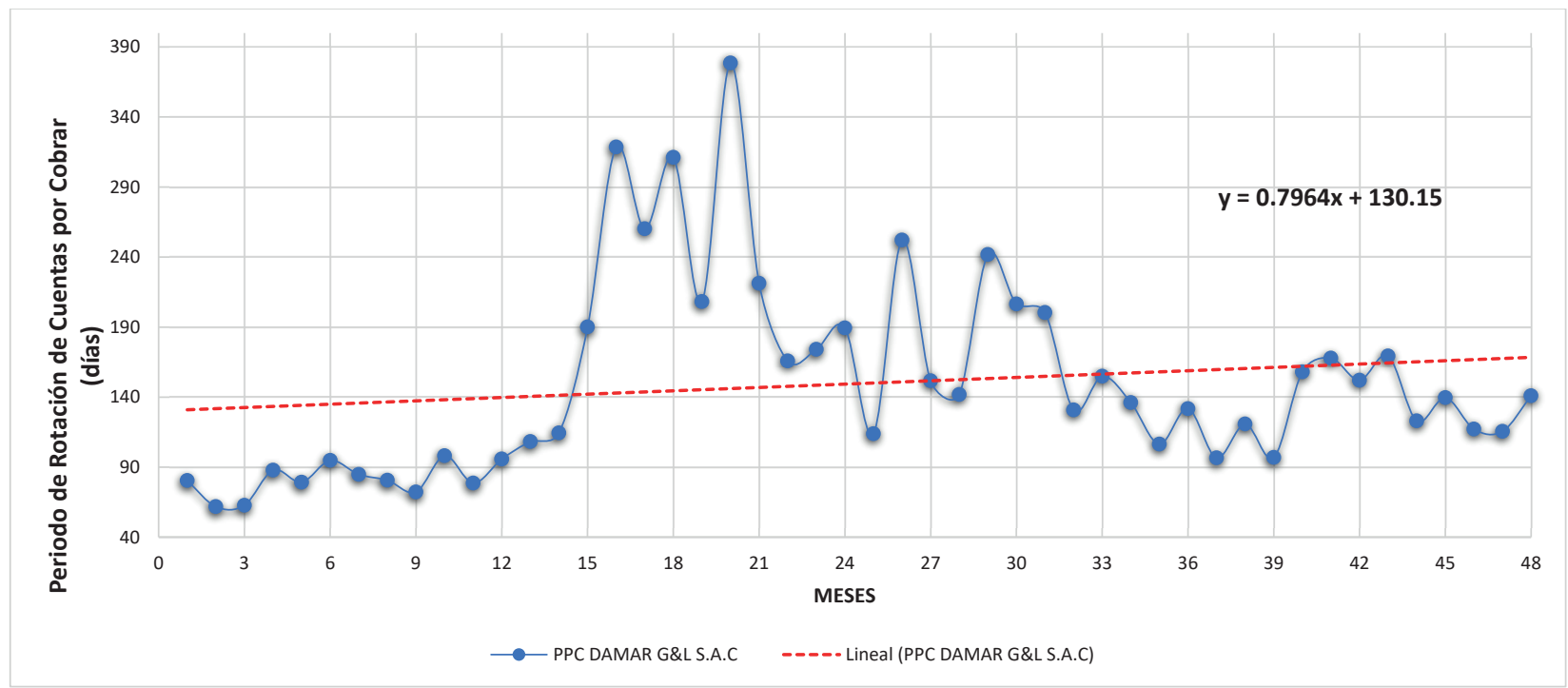

Figura 1. Comportamiento del periodo de rotación de cuentas por cobrar 2016-2019

Fuente: Elaboración propia 
año 2019 desciende a 169.36 días de cobro; estos niveles impactaron en la tendencia creciente del indicador durante los periodos de estudio, lo que indica que la empresa no ha mantenido una correcta política en los créditos otorgados a sus clientes.

En la figura 2 se muestra el comportamiento del indicador periodo de rotación de inventarios de la empresa industrial en los periodos de estudio 2016-2019, en donde se evidencia una pendiente negativa de -0.6827 , lo que refleja un nivel decreciente en el promedio de días en el que los inventarios son convertidos en productos terminados y vendidos a los clientes. En la variabilidad del indicador, se observa que en el año 2016 obtuvo un nivel más alto con un promedio de 79.26 días de inventario, pero a partir del mes de septiembre del año 2017 los días de inventario disminuyeron notablemente con un promedio de 17.22 días, al igual que en el año 2018, donde se obtuvo un promedio de 12.63 días y ya en el año 2019 el periodo promedio mínimo aumentó en 20.27 días; es por ello que, los niveles descendientes en los días promedio de inventario influyen en la tendencia negativa de la empresa en estudio, por lo que se determina que la empresa ha mantenido una mayor rotación en los días de inventario entre los años 2017 y 2018, produciendo y vendiendo en un menor tiempo.

El comportamiento de indicador periodo de rotación de cuentas por pagar entre los años de estudio 2016 al 2019 se aprecia en la figura 3. Se observa que la empresa industrial en estudio presenta una pendiente positiva de 1.2176, evidenciando un nivel de crecimiento en el promedio de días de pago. A pesar que, el promedio más bajo en los días de pago que se presentó en el año 2018 fue de 35.05 días, la empresa tuvo niveles crecientes en los periodos promedio de pago en los años 2017 y 2019, pues los promedios de días de pago fueron de 509.57 y 628.82 respectivamente; es por ello que, los niveles crecientes de los días de pago inciden de manera directa en la tendencia positiva del indicador durante los periodos 2016 al 2019, lo que a su vez demuestra, que la empresa no controla eficientemente los periodos de pago a causa de la falta de políticas de pago a proveedores, generando que la imagen de la empresa se pueda perjudicar por el incumplimiento de las deudas.

El comportamiento del ciclo de conversión del efectivo de la empresa Damar G\&L S.A.C. durante el periodo de estudio 2016 al 2019 se muestra en el grafico 4, en donde se observa una pendiente negativa de -1.1038 , evidenciando un descenso del ciclo de conversión del efectivo. Ahora bien, la tendencia de un ciclo de efectivo negativo demuestra que la empresa estaría pagando a sus proveedores después de haber cobrado por la venta de sus productos; resaltando que el periodo medio en el cual retorna el efectivo a la empresa es de 29 días, es en el año 2016 en donde la tendencia de un ciclo de efectivo menor es visible y con mayor notoriedad en el año 2019; lo que demuestra, que los periodos de mayor rotación de cuentas por cobrar y el incremento en los días de pago a los proveedores han influenciado en la tendencia negativa del ciclo de conversión del efectivo, reflejando una mejora en la recuperación de la inversión.

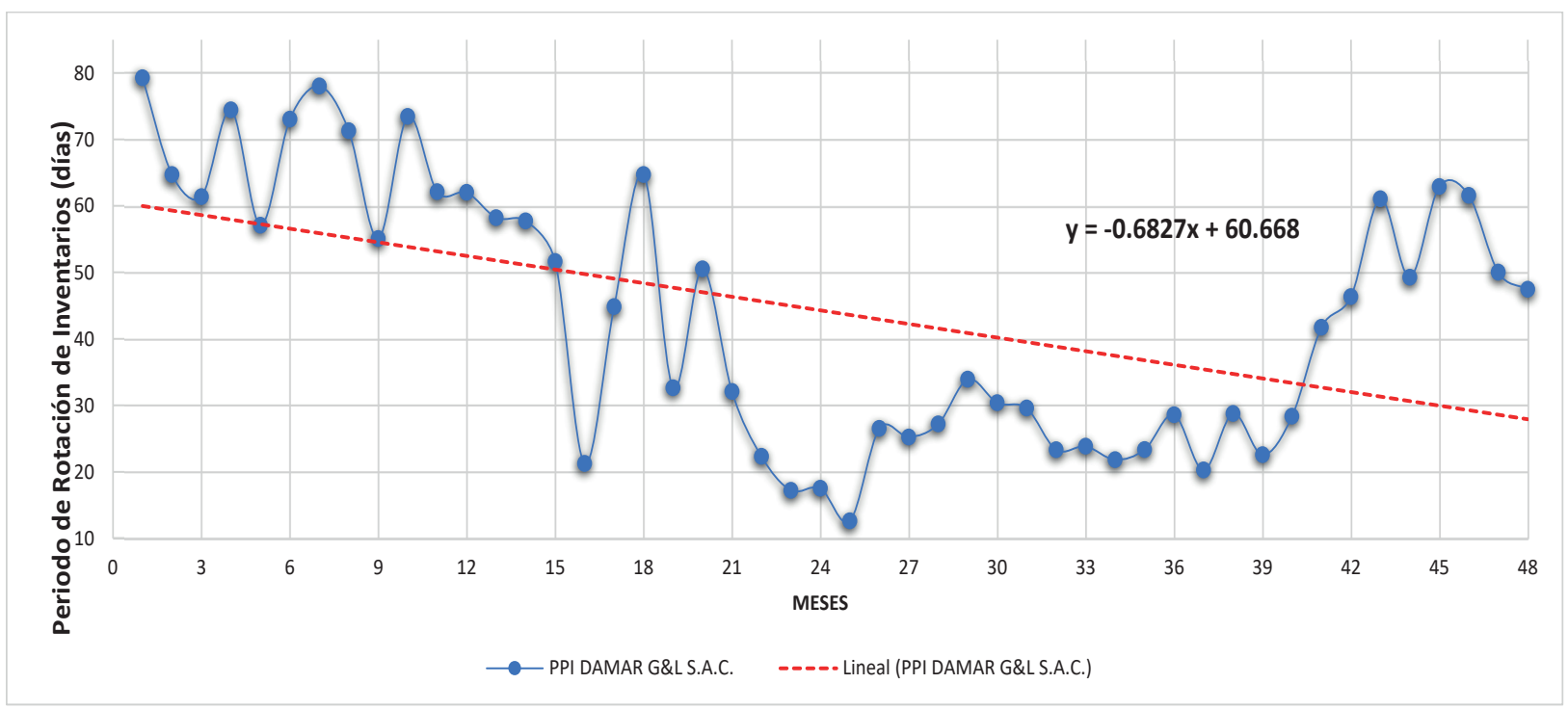

Figura 2. Comportamiento del periodo de rotación de inventarios 2016-2019

Fuente: Elaboración propia 


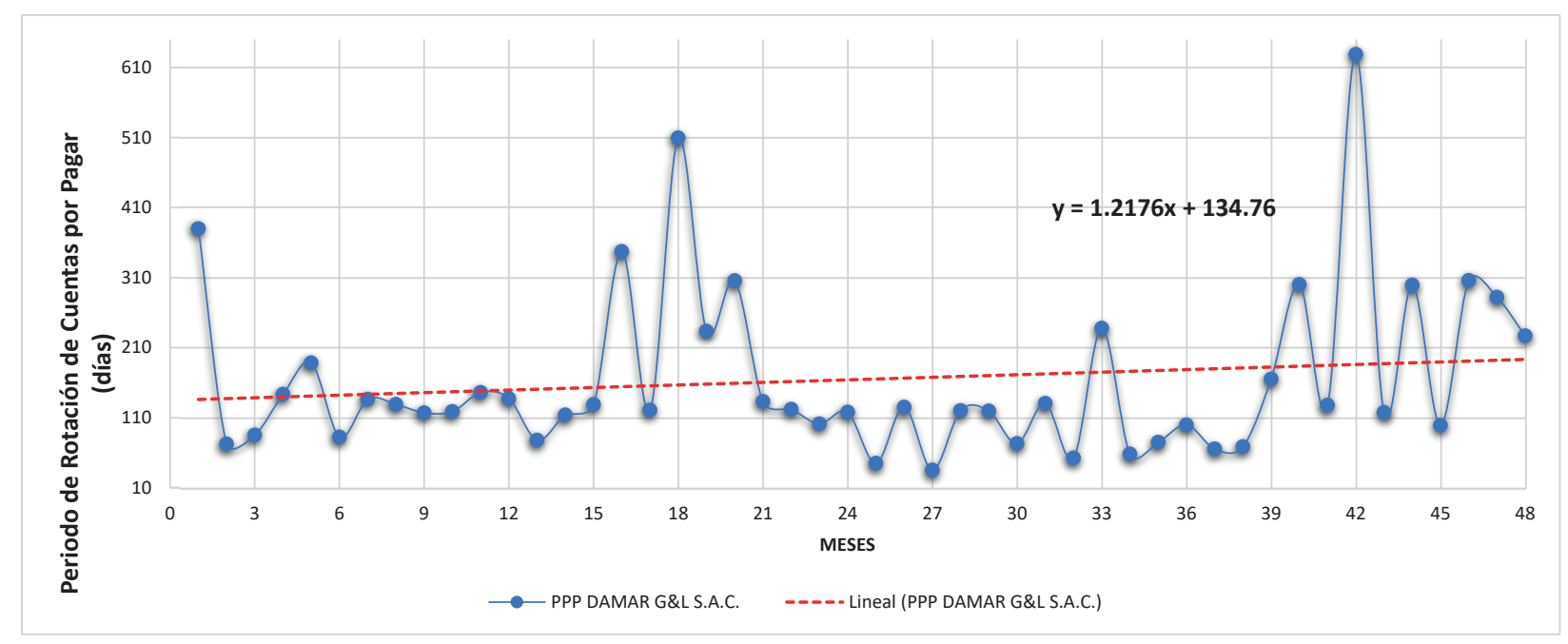

Figura 3. Comportamiento del periodo de rotación de cuentas por pagar 2016-2019

Fuente: Elaboración propia

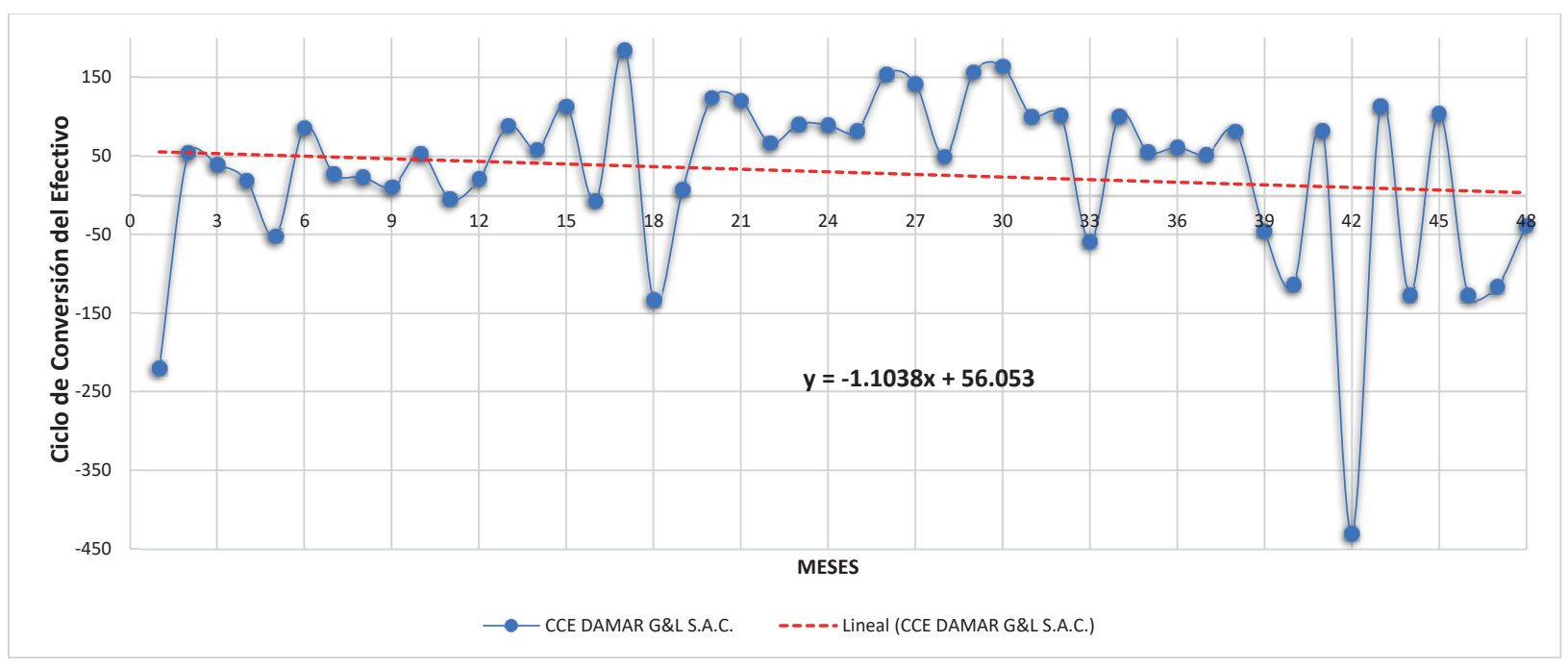

Figura 4. Comportamiento del ciclo de conversión del efectivo 2016-2019

Fuente: Elaboración propia

En la figura 5, se muestra el comportamiento de la prueba ácida de la empresa industrial durante el periodo de estudio 2016 al 2019. Se observa una pendiente mínimamente positiva de 0.0005 , reflejando un nivel promedio de 0.80 en el tiempo; presentando solo en los últimos y primeros meses de cada año incrementos en la liquidez, pues en diciembre 2016 alcanzó un 0.93, en diciembre 2017 logró un 1.08 , en enero y febrero 2018 alcanzó un 0.87 y solo en abril 2019 obtuvo 0.87 , a causa del incremento de la producción y el aprovechamiento de mayor demanda en las ventas (temporadas altas); demostrando que, a pesar de la existencia de niveles altos de liquidez, la empresa aún debe ser eficiente en la gestión de sus activos y pasivos para cubrir sus obligaciones de corto plazo durante todo el año.

En referencia al análisis inferencial para determinar el grado de relación del ciclo de conversión del efectivo en la liquidez de la empresa en estudio Damar G\&L S.A.C., durante los periodos 2016 al 2019; se realizó el contraste de hipótesis haciendo uso del coeficiente de correlación de Rho de Spearman, de acuerdo al análisis de normalidad de las variables. Asimismo, para el contraste de la hipótesis, 
se consideró el criterio del p-valor para aceptar o no aceptar la hipótesis nula. Estos criterios son los siguientes:

Si p-valor es $>\mathbf{0 . 0 5}$, se acepta la hipótesis nula y se rechaza la hipótesis alterna.

Si p-valor es $\leq \mathbf{0 . 0 5}$, se rechaza la hipótesis nula y se acepta la hipótesis alterna.

En la tabla 3, se muestra el análisis de la correlación de las variables de estudio. En cuanto a la relación entre el periodo de rotación de cuentas por cobrar y la prueba ácida, se encontró que no existe una relación estadísticamente significancia según p-valor $=.169>.05$; por tanto, se determina que si los días promedio de cobro disminuyen no influirán directamente en prueba ácida. En relación con el periodo de rotación de inventarios y la prueba ácida, se evidencia la inexistencia de una relación estadísticamente significativa $\mathrm{p}$-valor $=.063>.05$; por consiguiente, se establece que si los días promedio de inventarios disminuyen no incidirán de manera directa en la prueba ácida. En cuanto al grado de relación entre el periodo de rotación de cuentas por pagar y la prueba ácida, se observa que no existe una relación estadísticamente significativa según p-valor $=.559>.05$; confirmando que, en la medida que los días promedio de pago se incrementen no repercutirán del mismo modo en la prueba ácida. De igual manera, los resultados obtenidos en relación con el ciclo de conversión del efectivo y la liquidez, se determina que no existe una relación estadísticamente significativa ( $\mathrm{p}$-valor $=.614>$ .05); determinando que, en la medida que el ciclo de conversión del efectivo se incremente o disminuya no influirá de igual forma en la liquidez de la empresa industrial.

\section{DISCUSIÓN}

La administración del efectivo es muy importante en las empresas, por lo que el área de finanzas debe trabajar de manera eficaz para mantener un constante equilibrio tanto en los activos corrientes como en los pasivos corrientes, puesto que estos reflejan la liquidez empresarial

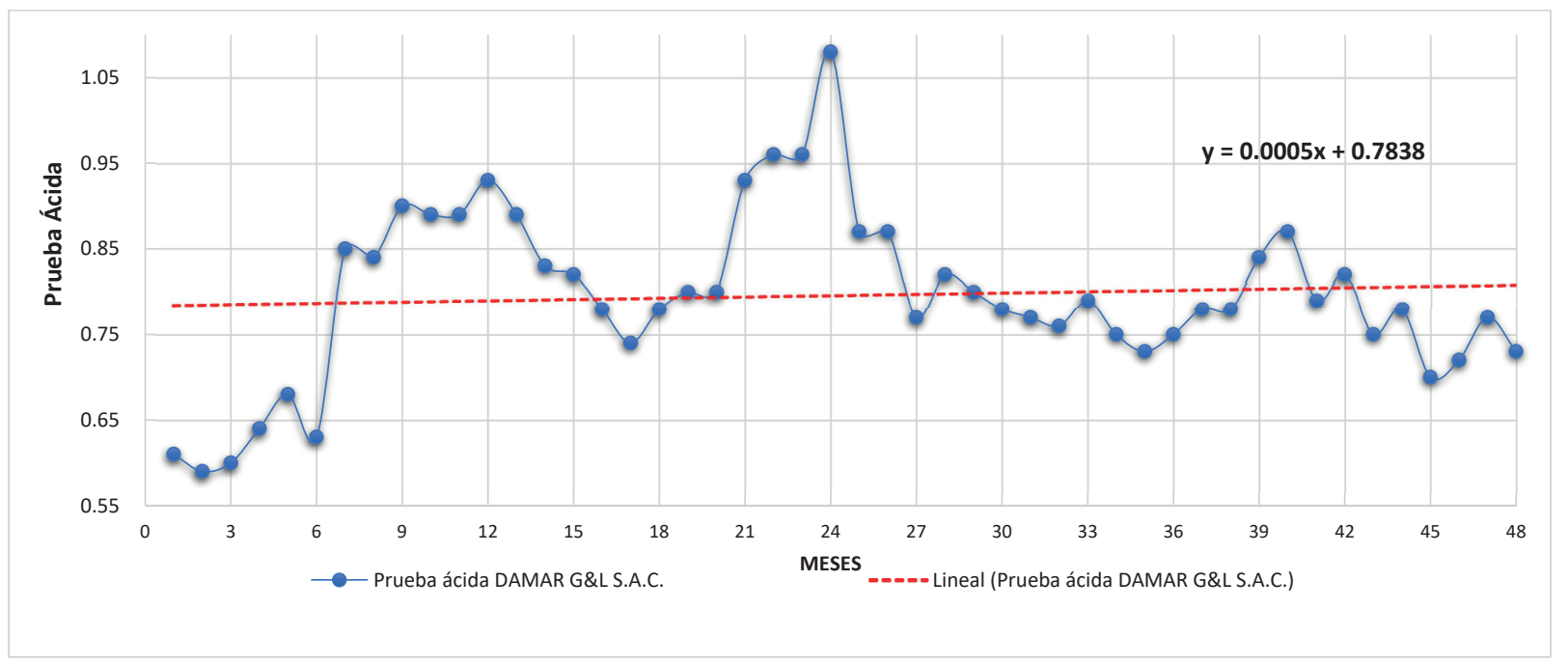

Figura 5. Comportamiento de la prueba ácida 2016-2019

Fuente: Elaboración propia

Tabla 3

Resultados del análisis de correlación

\begin{tabular}{lccc}
\hline & \multicolumn{2}{c}{ Prueba ácida } & $\mathbf{N}$ \\
\cline { 2 - 4 } & Rho de Spearman & $\mathbf{p}$ & 48 \\
Periodo de rotación de cuentas por cobrar & .202 & .169 & 48 \\
Periodo de rotación de inventarios & -.270 & .063 & .559 \\
Periodo de rotación de cuentas por pagar & .086 & .614 & 48 \\
Ciclo de conversión del efectivo & .075 & 48 \\
\hline
\end{tabular}

Fuente: Elaboración propia 
al sostener una gestión firme y coherente de las políticas aplicadas, referente a las cuentas por cobrar, inventarios y cuentas por pagar.

Los resultados de la investigación evidencian que, el ciclo de conversión del efectivo y la liquidez de la empresa industrial Damar G\&L S.A.C. durante los periodos 2016 al 2019, no muestran una relación estadísticamente significativa $(\mathrm{Rho}=.075, \mathrm{p}=.614)$. Por ello se concluye que, en la medida que el ciclo de conversión del efectivo incremente o disminuya, este no se reflejará de manera directa en la liquidez de la empresa industrial; asimismo, los indicadores periodo de rotación de cuentas por cobrar, periodo de rotación de inventarios y periodo de rotación de cuentas por pagar muestran una relación débil en la prueba ácida, pues el nivel de significancia es mayor al $5 \%$. Por el contrario, las investigaciones realizadas por Graziano (2016) y Molina (2016) muestran que si existe una relación estadísticamente significativa entre el ciclo de conversión del efectivo y la liquidez de las empresas en estudio. Sin embargo, es importante reflejar que al relacionar las variables, se emplean porcentajes basados en encuestas y cuestionarios que no obedecen a la naturaleza de las variables, puesto que el tratamiento indica que los datos deben ser recabados desde los registros contables y balances generales. De esta manera, al demostrar que las investigaciones no aplican ratios financieros para medir relación, se entiende que los resultados obtenidos en el presente estudio se acercan más a la realidad de la empresa $y$, por ende, favorecen en la toma de decisiones por parte de la gerencia.

Por otra parte, al analizar el comportamiento de los indicadores de las variables de estudio, se puede explicar las variaciones cambiantes como una consecuencia de no tener determinados correctamente los días de rotación de los activos y pasivos corrientes, pues al no existir tratos consistentes con los clientes para el reembolso del dinero, muchos de ellos aprovechan el pago a largos plazos, lo que a su vez genera una inexactitud del periodo de cobro. Asimismo, los periodos largos de inventarios necesitan de una consistente proyección de ventas, con el fin de generar que la inversión retorne más rápido y así originar oportunidades de mercado; además, los periodos de pago deben ser equilibrados siempre tomando en cuenta los reembolsos obtenidos de los clientes, y aprovechando los descuentos concedidos por pronto pago, teniendo cuidado de no perder la imagen de buen contribuyente. De esta misma manera lo indica el estudio hecho por Castro (2017), en donde refiere que la implementación de políticas de inventarios, cobranzas y pago, optimizará las herramientas respecto a la gestión de los inventarios, permitiendo controlar aquellas existencias que rotan mucho más rápido, pero sin descuidar a las que tiene menor rotación; además, ayudará a implementar y controlar nuevas formas de cobro a los clientes para que este se realice en un plazo mucho menor, con la finalidad de tener suficiente capital propio y se tenga la capacidad de solventar los pagos a los proveedores.

Finalmente, en el análisis de los indicadores financieros se ha determinado que, durante los 48 meses de estudio, la empresa Damar G\&L S.A.C tiene un ciclo operativo mayor al ciclo de pago; por ello, es recomendable y necesario que pueda implementar y equilibrar las políticas existentes de cobro, inventario y de pago a las necesidades y condiciones del mercado empresarial actual.

\section{REFERENCIAS}

Al-Shubiri, F., y Mohammad, N. (2013). The relationship between cash conversion cycle and financial characteristics of industrial sectors: an empirical study. Investment Management and Financial Innovations, 10(4), 95-102. Recuperado de: https://www.researchgate.net/ publication/286135697_The_relationship_between_ cash_conversion_cycle_and_financial_characteristics_of_industrial_sectors_An_empirical_study

Arcos, M., y Benavides, J. (2008). Efecto del ciclo de efectivo sobre la rentabilidad de las firmas colombianas. Cuadernos de Administración, 21(36), 167-182. Recuperado de: http://www.scielo.org.co/pdf/cadm/ v21n36/v21n36a08.pdf

Castro, K. (2017). Ciclo de conversión del efectivo y la liquidez de la empresa Electro Frío (Tesis de pregrado). Universidad Laica Vicente Rocafuerte de Guayaquil, Guayaquil. Recuperado de: http://repositorio.ulvr.edu. ec/handle/44000/1564

Culqui, L. (2011). El ciclo de caja y su incidencia en la liquidez de Construcciones Civil-Hidráulica Culqui Ninacuri en el periodo 2008-2010 (Tesis de pregrado). Universidad Técnica de Ambato, Ecuador. Recuperado de: https://repositorio.uta.edu.ec/jspui/handle/123456789/1970

García, E. (8 de diciembre de 2018). Compañías locales son más líquidas que las de Chile y México. Diario Gestión. Recuperado de: https://gestion.pe/econo$\mathrm{mia} /$ companias-locales-son-liquidas-chile-mexico-252198-noticia/

García, E. (12 de mayo de 2019). Riesgo de liquidez de empresas peruanas es elevado pero menor que el de hace dos años. Diario Gestión. Recuperado de: https://gestion.pe/economia/riesgo-liquidez-empresas-peruanas-elevado-menor-dos-anos-266536-noticia/?ref=gesr 
García, J., Galarza, S., y Altamirano, A. (2017). Importancia de la administración eficiente del capital de trabajo en las Pymes. Revista Ciencia UNEMI, 10(23), 30-39. Recuperado de: http://ojs.unemi.edu.ec/index.php/ cienciaunemi/article/view/495

Gitman, L., y Zutter, C. (2012). Principios de administración financiera (12. ${ }^{\mathrm{a}}$ ed.). México D.F.: Pearson Education. Recuperado de: https://www.academia. edu/28949029/LIBRO_Principios_de_Administracion_Financiera_LAWRENCE_J._GITMAN

Graziano, M. (2016). Ciclo de conversión del efectivo en la inversión y liquidez en las empresas picadoras de piedra caliza del Municipio Rosario de Perijá (Tesis de pregrado). Universidad Rafael Urdaneta, Maracaibo. Recuperado de: https://www.academia.edu/30027231/ CICLO_CONVERSION_DEL_EFECTIVO_pdf

Hernández, R., Fernández, C., y Baptista, M. (2014). Metodología de la investigación (6.a ed., Vol. 3). México D.F.: McGraw-Hill/Interamericana Editores S.A. de C.V. Recuperado de: https://periodicooficial.jalisco. gob.mx/sites/periodicooficial.jalisco.gob.mx/files/ metodologia_de_la_investigacion_-_roberto_hernandez_sampieri.pdf

Jiménez, J., Rojas, F., y Ospina, H. (2013). La importancia del ciclo de caja y cálculo del capital de trabajo en la gerencia PYME. Clío América, 7(13), 48-63. Recuperado de: https://dialnet.unirioja.es/servlet/articulo?codigo $=5114823$

Lyroudi, K., y Mccarty, D. (1993). An Empirical Investigation of the Cash Conversion Cycle of Small Business Firms. Journal of Small Business Finance, 2(2), 139161. Recuperado de: https://digitalcommons.pepperdine.edu/jef/vol2/iss2/4/

Norma Internacional de Contabilidad $\mathrm{N}^{\circ}$ 1. (2006). Presentación de Estados Financieros. Recuperado de: https://www.nicniif.org/files/NIC\%201\%20Presentacion\%20de\%20Estados\%20Financieros.pdf
Molina, R. (2016). Ciclo de conversión del efectivo y su relación en la liquidez de la empresa Lácteos Piamonte S.A.C. en el período 2016 (Tesis de pregrado). Universidad César Vallejo, Trujillo. Recuperado de: https:// repositorio.ucv.edu.pe/handle/20.500.12692/3044

Mongrut, S., Fuenzalida, D., Cubillas, C., y Cubillas, J. (2014). Determinants of Working Capital Management in Latin American Companies. Innovar, 24(51), 5-18. Recuperado de: https://revistas.unal.edu.co/index.php/innovar/article/view/41235

Murtala, Z., y Sani, S. (2016). The Impact of Cash Conversion Cycle on Firm Profitability: Evidence from Nigerian Listed Telecommunication Companies. Journal of Finance and Accounting, 4(6), 342-350. Recuperado de: http://www.sciencepublishinggroup.com/ journal/paperinfo?journalid $=171 \&$ doi $=10.11648 /$ j jfa.20160406.15

Ramos, K., y Sandoval, W. (2019). Estudio de caso: análisis del Ciclo de Conversión del Efectivo - CCE (Tesis de pregrado). Universidad Católica de Colombia, Bogotá. Recuperado de: https://repository.ucatolica.edu.co/ handle/10983/23534

Ross, S., Westerfield, R., y Jordan, B. (2010). Fundamentos de finanzas corporativas (9. ${ }^{\mathrm{a}}$ ed.). México DF.: McGraw-Hill/Interamericana Editores, S.A. de C.V. Recuperado de: https://www.academia. edu/32709834/Fundamentos_de_Finanzas_Corporativas_-_Ross_9th

Samper, J. (23 de setiembre de 2015). Periodo medio de maduración (PMM). Economipedia.com. Recuperado de: https://economipedia.com/definiciones/periodo-medio-de-maduracion-pmm.html

Sanjines, X. (2019). Aplicación de las razones financieras de actividad en una organización: gestión y repercusiones en la liquidez (Tesis de pregrado). Universidad Técnica de Machala, Machala. Recuperado de: http://repositorio.utmachala.edu.ec/handle/48000/13694 